\title{
Factors controlling the molecular changes of dissolved organic matter in the Pearl River Estuary
}

\author{
Wei Xie ${ }^{1}$, Penghui Li, Ding He, Chen He, Quan Shi,
} CHUANLUN ZHANG ${ }^{2}$

1xiewei9@mail.sysu.edu.cn

${ }^{2}$ zhangcl@sustech.edu.cn

The Pearl River is the second largest river in Southern China, flowing through a highly populated and economically developed region, and discharging significant amount of terrestrial organic carbon into the South China Sea through the Pearl River Estuary (PRE) where active primary production forms new biomass into the organic carbon pool. Thus disentangling the composition of organic matter in estuaries has been challenging. In this study, the molecular composition of solid-phase extracted (SPE) dissolved organic matter (DOM) along PRE gradient were analyzed by negative-ion electrospray ionization (ESI) Fourier transform ion cyclotron resonance mass spectrometry (FT-ICR MS). The results from SPE-DOM exhibited a total of 13,933 unique molecular formulae from 87 samples from PRE with salinity ranging from 0 to 32.6. According to their $\mathrm{O} / \mathrm{C}$ and $\mathrm{H} / \mathrm{C}$ ratios, those molecules were assigned to classes belonging to highly unsaturated compounds $\mathrm{s}(82.6+3.2 \%)$, carboxyl-rich alicyclic molecules $(68.0+2.0 \%)$, polyphenols $(5.4+1.5 \%)$, unsaturated aliphatic compound $(8.9+1.6 \%)$ and peptides $(0.3+0.18 \%)$. Among environmental parameters, salinity was found to be the major factor controlling the distribution of those molecule classes, with the highly unsaturated compounds being positively correlated with salinity $(\mathrm{R} 2=0.73)$ and polyphenols negatively correlated with salinity $(\mathrm{R} 2=0.93)$. These results indicate unsaturated compounds may be formed in situ in the water column while polyphenols represent terrestrial material resisting degradation in PRE. A bio-degradation experiment over 28 days in the laboratory also showed the less degradation of those polyphenols. Collectively, our study demonstrated that polyphenols were terrestrially derived and biorefractory, which may serve as a biomarker for terrestrial input of dissolved organic matter in PRE. 